\title{
Bacterial endocarditis masked by COVID-19: A case report
}

\author{
MIRCEA BAJDECHI ${ }^{1}$, NICOLETA DORINA VLAD ${ }^{1}$, MIRELA DUMITRASCU ${ }^{2}$, ELENA MOCANU ${ }^{3 *}$, \\ IRINA MAGDALENA DUMITRU ${ }^{1,2,4 *}$, ROXANA CARMEN CERNAT ${ }^{2,4^{*}}$ and SORIN RUGINA $\breve{~}^{1,2,4}$ \\ ${ }^{1}$ Doctoral School of Medicine, 'Ovidius' University, 900470 Constanta; ${ }^{2}$ Department of Infectious Disease, \\ Clinical Infectious Diseases Hospital, 900709 Constanta; Departments of ${ }^{3}$ Public Health and ${ }^{4}$ Infectious Disease, \\ Faculty of Medicine, 'Ovidius' University, 900470 Constanta, Romania
}

Received September 15, 2021; Accepted October 15, 2021

DOI: $10.3892 / \mathrm{etm} .2021 .11109$

\begin{abstract}
Infective endocarditis represents a rare complication among patients infected with severe acute respiratory syndrome coronavirus-2 (SARS-CoV-2); it is often a nosocomial infection and the symptomatology can be masked by respiratory failure symptoms from SARS-CoV-2 bronchopneumonia. Management of patients with severe forms of SARS-COV-2 infection who also have associated infective endocarditis is very difficult, especially in mono-specialty hospitals (such as infectious diseases hospitals) where access to cardiological investigations is limited. The current study presents the case of a 73-year-old woman with increased cardiovascular risk (high blood pressure, diabetes mellitus and obesity), with uninvestigated ischaemic heart disease, who was admitted to the Department of Infectious Diseases in the Clinical Infectious Diseases Hospital (Constanta, Romania) due to SARS-CoV-2. Although the evolution was initially favorable, the condition of the patient significantly deteriorated on the 14th day of hospitalization due to the development of Enterococcus faecium infective endocarditis. Despite the therapy, the evolution was fulminant. Infection with coronavirus disease 2019 can result in numerous comorbidities, which cause higher mortality rates than in the general population.
\end{abstract}

\section{Introduction}

Pre-existing cardiovascular comorbidities are important risk factors for patients with severe acute respiratory syndrome coronavirus-2 (SARS-CoV-2). A recent study reported a 20 -fold higher mortality rate in patients with cardiovascular

Correspondence to: Dr Mircea Bajdechi, Doctoral School of Medicine, 'Ovidius' University, 1 University Street, 900470 Constanta, Romania

E-mail: mircea.bajdechi@gmail.com

*Contributed equally

Key words: coronavirus disease 2019, severe acute respiratory syndrome coronavirus-2, positive blood culture, Enterococcus faecium, infective endocarditis diseases and SARS-CoV-2 infection, with a $>10$ times higher risk of being admitted to the intensive care unit (1). Heart failure, coronary artery disease and arrhythmias are risk factors for coronavirus disease 2019 (COVID-19) progression and they are associated with poor outcome (2).

However, one study showed that acute cardiovascular events during COVID-19 hospitalization are frequent. Among the 3,011 hospitalized patients from The Netherlands over a 3 -month period, cardiac complications occurred in $11.6 \%$, atrial fibrillation being the most common, followed by heart failure, acute coronary syndrome, ventricular arrhythmia, bacterial endocarditis, myocarditis, pericarditis and pulmonary embolism (3).

During the pandemic, a total of 920 COVID-19 patients were admitted to the Department of Infectious Disease in the Clinical Infectious Diseases Hospital (Constanta, Romania) between March 2020 and March 2021. The patients exhibited different stages of the disease, with most of them having moderate or severe forms. Cardiovascular complications were diagnosed in 103 patients (11.20\%), with cardiac rhythm and conduction abnormalities being the most frequent, including atrial fibrillation $(n=12)$, atrial flutter $(n=2)$, other supraventricular tachycardias $(\mathrm{n}=19)$, bradycardia $(\mathrm{n}=16)$, bundle branch block $(n=6)$ and a prolonged QT interval $(n=16)$. The incidence of pulmonary embolism was $0.87 \%(\mathrm{n}=8)$ and of deep vein thrombosis $0.65 \%(n=6)$. Acute renal artery occlusion caused by systemic thromboembolism occurred in two cases. The incidence of other cardiovascular complications was $1.20 \%$ for pericarditis $(n=11), 0.33 \%$ for stroke $(n=3)$, $0.11 \%$ for myocarditis $(\mathrm{n}=1)$ and $0.11 \%$ for bacterial endocarditis $(n=1)$. Most of the complications occurred in men, with a median age of 69 years, male sex and advanced age ( $>55$ years old) being two important cardiovascular factors. SARS-COV2-infected patients have a high risk of developing cardiovascular complications such as bacterial endocarditis. A complete routine echocardiography, in patients with severe SARS-COV-2 pneumonia admitted to intensive care units may be required for the early diagnosis and treatment of infective endocarditis.

\section{Case report}

A 73-year-old woman was admitted in May 2021 to the Department of Infectious Disease in the Clinical Infectious 
Diseases Hospital with a cough, shortness of breath and fatigue that was found to be due to severe SARS-CoV-2 pneumonia by nasopharyngeal swab. The patient had multiple cardiovascular risk factors, including arterial hypertension, type 2 diabetes mellitus, obesity (36.7 BMI) and a history of coronary heart disease for a number of years. Chest X-rays showed bilateral pneumonia with peripheral consolidations and a reticular pattern due to septal thickening with ground-glass opacities (Fig. 1). From admission, the patient exhibited dyspnea, with acute respiratory failure (mixed venous oxygen saturation, $78 \%$ ), and blood tests showed inflammatory syndrome [elevated erythrocyte sedimentation rate $(80 \mathrm{~mm} / \mathrm{h}$; cut-off, $3-9 \mathrm{~mm} / \mathrm{h})$, and fibrinogen $(820 \mathrm{mg} / \mathrm{dl}$; cut-off, 196-372 mg/dl), C reactive protein $(120 \mathrm{mg} / \mathrm{l}$; cut-off, $0-5 \mathrm{mg} / \mathrm{dl})$, ferritin $(1,206 \mathrm{ng} / \mathrm{ml}$; cut-off, $70-435 \mathrm{ng} / \mathrm{ml})$ and IL-6 (276 pg/ml; cut-off, $0-7 \mathrm{pg} / \mathrm{ml})$ levels]. The serum level of procalcitonin was normal. The patient received corticosteroid therapy ( $8 \mathrm{mg}$ dexamethasone twice daily) from admission, as well as tocilizumab (2x800 $\mathrm{mg}$ twice daily), remdesivir ( $200 \mathrm{mg}$ on day 1 , then $100 \mathrm{mg}$ daily for 5 days) and prophylactic low molecular weight heparin (4,000 IU enoxaparin daily). The clinical and biological evolution was favorable during the first hospitalization period, but on the 14th day, the patient developed a fever $\left(38.5^{\circ} \mathrm{C}\right.$ temperature $)$ and their general condition worsened. Laboratory tests revealed a high procalcitonin level of $4.1 \mathrm{ng} / \mathrm{ml}$ (cut-off $<0.5 \mathrm{ng} / \mathrm{ml}$ ), mildly elevated liver transaminase levels (ALT, $120 \mathrm{u} / \mathrm{l}$; cut-off, 4-36 U/1; AST, $163 \mathrm{U} / 1$; cut-off, 5-40 U/1) and a decreased estimated glomerular filtration rate $\left(41 \mathrm{ml} / \mathrm{min} / 1.73 \mathrm{~m}^{2}\right)$. The hs-troponin (152 ng/l; cut-off, 4.9-50 ng/l) and D-Dimer (1.78 $\mu \mathrm{g} \mathrm{FEU/ml;} \mathrm{cut-off,} \mathrm{0-0.5} \mu \mathrm{g} \mathrm{FEU/ml)} \mathrm{levels} \mathrm{were} \mathrm{also}$ elevated. The patient developed respiratory distress (arterial oxygen partial pressure/fractional inspired oxygen ratio $<100)$ that required non-invasive continuous positive airway pressure ventilation. A pulmonary computed tomography scan was therefore performed, which ruled out a pulmonary embolism and showed diffuse ground-glass opacities, peripheral consolidation and bronchiectasis (Fig. 2). Blood cultures were positive for Enterococcus faecium sensible to vancomycin, linezolid, teicoplanin and tigecycline.

A transthoracic echocardiography was performed and revealed vegetation on the posterior mitral valve leaflet measuring $14 \times 5 \mathrm{~mm}$, leading to moderate mitral regurgitation (Fig. 3). Infective endocarditis was defined by the modified Duke criteria (4). A positive diagnosis of infective endocarditis manifesting $>48 \mathrm{~h}$ after hospital admission was based on a positive Enterococcus faecium blood culture and posterior mitral valve vegetation revealed by transthoracic echocardiography.

Antibiotic therapy with $1,000 \mathrm{mg}$ meropenem (3 times daily) and $600 \mathrm{mg}$ linezolid (2 times daily) was administered. Initially the patient had a favorable evolution, with improvements in symptomatology and inflammatory syndrome, but after 20 days of treatment, thrombocytopenia (platelet count, 58,000/ $\mathrm{mm}^{3}$ ) developed. Thus, linezolid was switched to teicoplanin $(12 \mathrm{mg} / \mathrm{kg}$ body weight twice daily as the loading dose, followed by $12 \mathrm{mg} / \mathrm{kg}$ body weight daily for the maintenance dose).

Despite antibiotic treatment, the patient developed multiple organ system failure and died 25 days after admission.

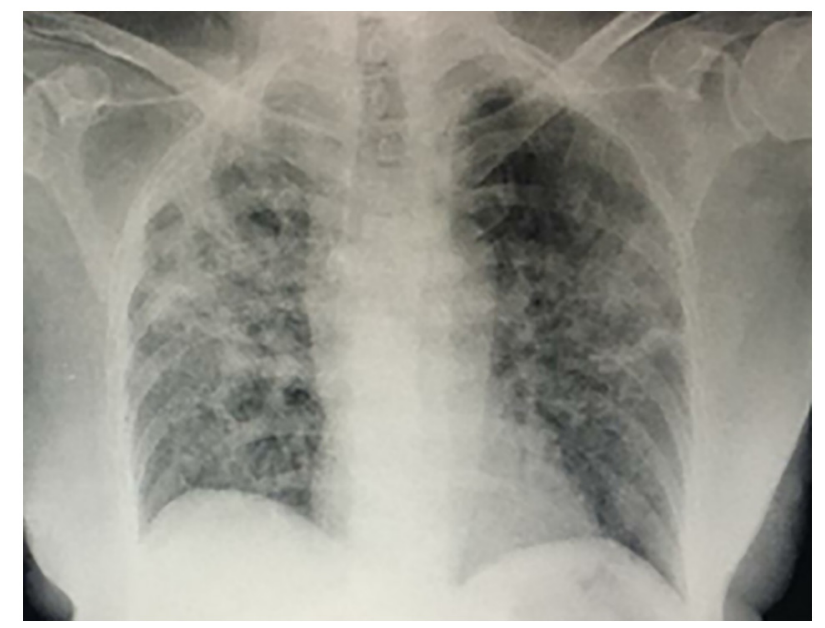

Figure 1. Chest X-ray image showing bilateral pneumonia with peripheral consolidations and a reticular pattern due to septal thickening, with ground-glass opacities.

\section{Discussion}

Bacterial endocarditis is a less frequent disease among patients infected with SARS-CoV-2 compared to other cardiac comorbidities in these patients. However, a study published by Ramos-Martínez et al (5) showed a higher incidence of hospital-acquired infective endocarditis in a single hospital during the first 2 months of the COVID-19 pandemic. Compared with the pre-pandemic period, when the incidence was 0.3 patients/month, during March and April 2020, the incidence was 2 patients/month $(\mathrm{P}=0.033)$. The etiology of infective endocarditis was Enterococcus faecalis (2 cases), Staphylococcus aureus (1 case) and Candida albicans (1 case), with an in-hospital mortality rate of $50 \%$.

It has also been observed that the etiology of endocarditis associated with SARS-COV-2 infection is different from the etiology of endocarditis in patients without SARS-COV-2 infection. Erdem et al (6) performed a recent study on 867 patients diagnosed with bacterial endocarditis and the most frequently involved germ was found to be Staphylococcus aureus, followed by Streptococcus viridans and Enterococci (untyped, E. Faecium and E. Faecalis). Endocarditis can be overlooked in patients with COVID-19, as SARS-CoV-2, acute respiratory distress syndrome and inflammatory syndrome can mask the symptoms. Still, the reported endocarditis cases revealed gram-negative germs, especially Klebsiella pneumoniae and Enterococcus faecium $(7,8)$.

Echocardiography is the most useful imaging technique to diagnose endocarditis due to its large availability, accuracy and safety, allowing a comprehensive evaluation of cardiac valves (9).

In the present study, the patient exhibited the risk factors of advanced age, pre-existing cardiac comorbidities, type 2 diabetes mellitus, obesity, immunosuppressive therapy and central venous catheterization upon admission. Similar to patients with HIV, patients infected with COVID-19 can have multiple comorbidities, which cause higher mortality rates compared with that in the general population, in spite of therapeutic care advances (10). 

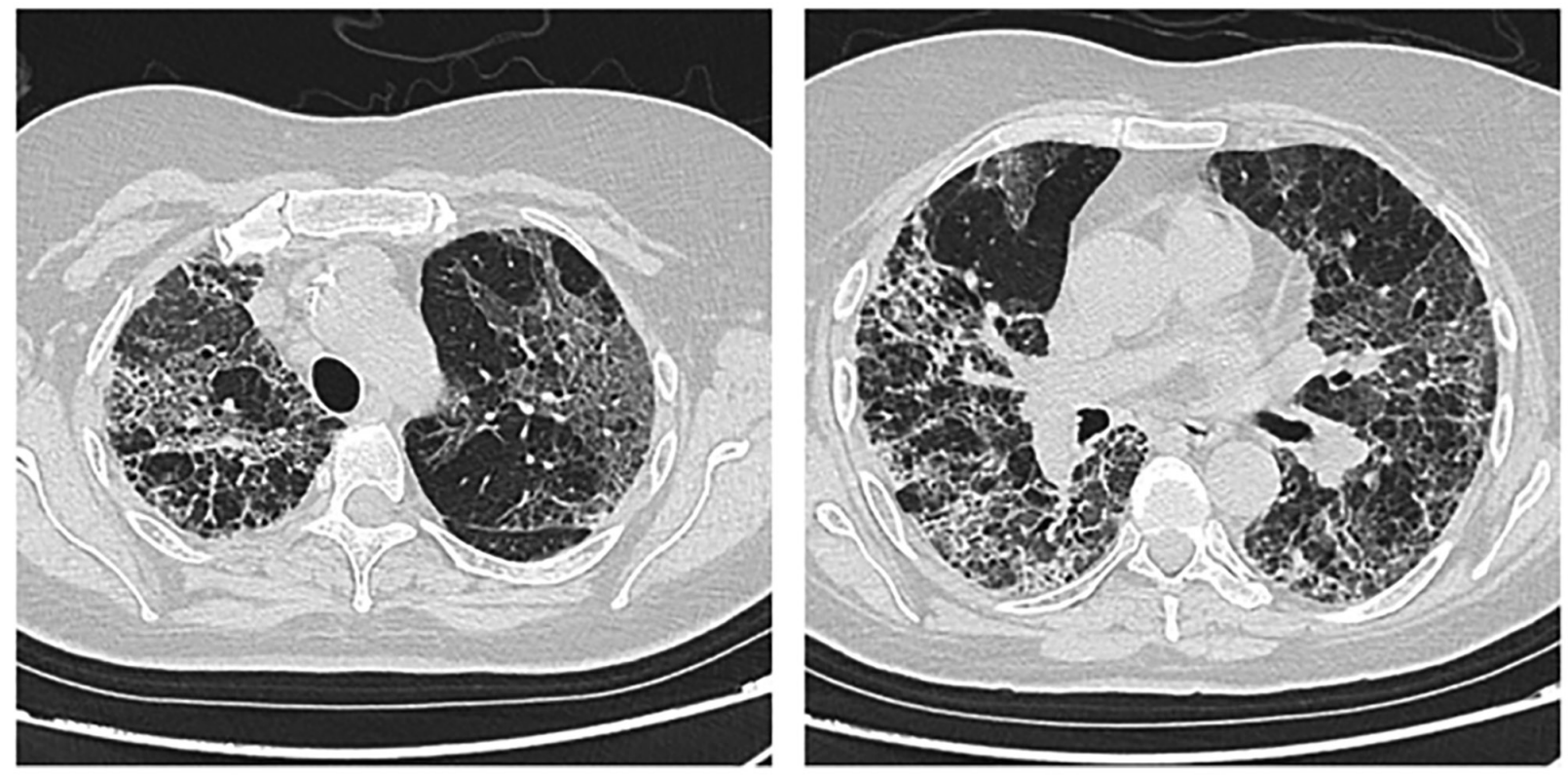

Figure 2. Computed tomography images showing diffuse ground-glass opacities, peripheral consolidation and bronchiectasis.

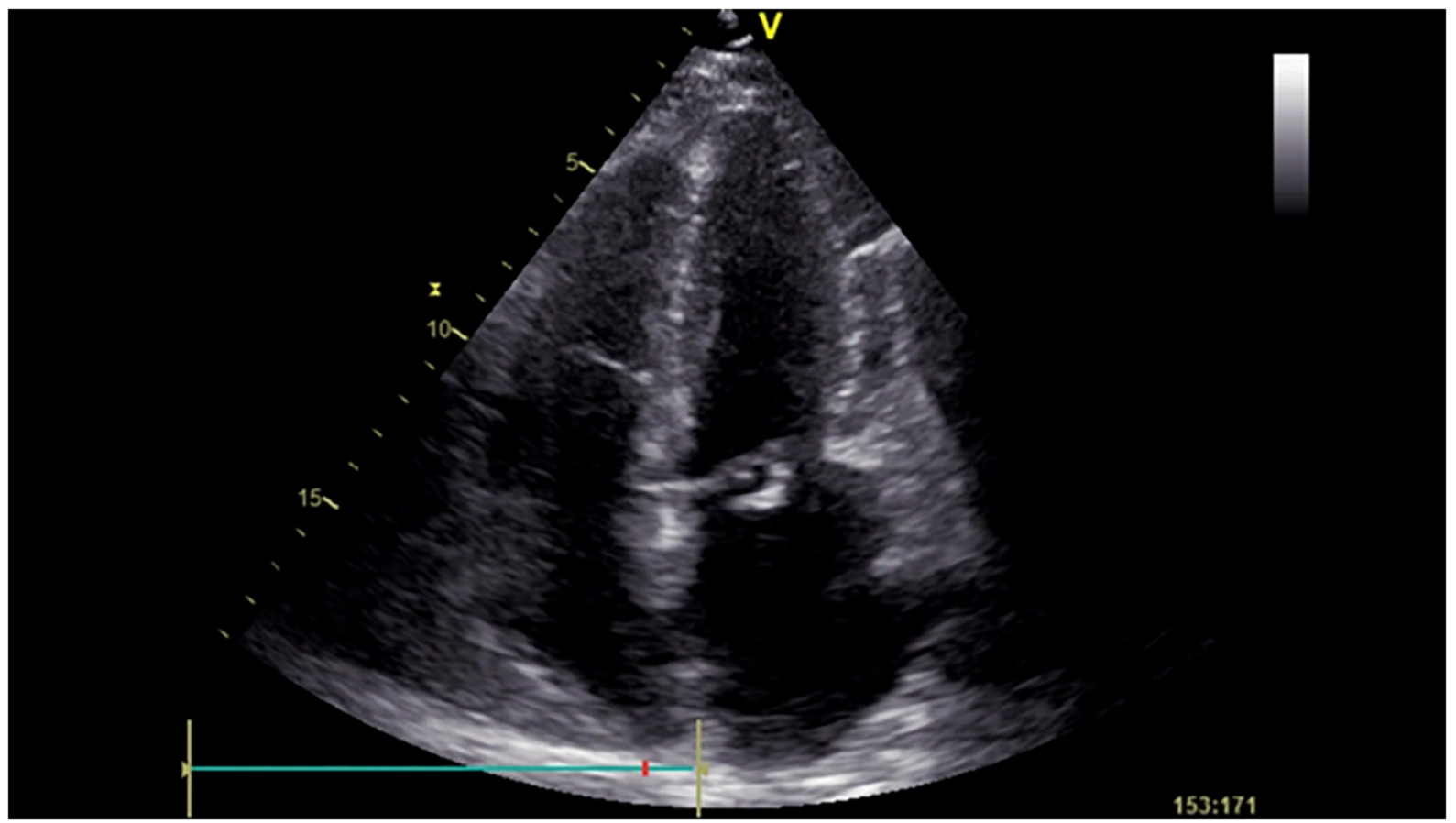

Figure 3. Transthoracic echocardiography (apical 4 chamber view) showing vegetation on the posterior mitral valve leaflet measuring $14 \times 5 \mathrm{~mm}$, with moderate mitral regurgitation.

The number and severity of respiratory infections is continuously increasing due to environmental changes and the lack of vaccination (11). The mRNA vaccine against SARS-COV-2, based on a new genetic method, turns the human body into living laboratory (12). Although vaccination represents the main weapon against the virus, prevention strategies such as early detection of infected individuals, updating new discoveries and social distancing still play a major role (13).

In conclusion, SARS-COV2-infected patients have a higher risk of developing cardiovascular complications. This viral infection is able to mask bacterial endocarditis. The management of patients with severe of SARS-COV-2 infection who also exhibit associated infectious endocarditis is difficult, especially in mono-specialty hospitals (such as infectious diseases hospitals) where access to cardiological investigations is limited. A complete routine examination, including echocardiography, in patients with severe SARS-COV-2 pneumonia admitted to intensive care units may be required for the early diagnosis and treatment of infective endocarditis. Bacterial endocarditis remains a major challenge in patients infected with SARS-COV-2, with a poor prognosis. 


\section{Acknowledgements}

Not applicable.

\section{Funding}

No funding was received.

\section{Availability of data and materials}

All data generated or analyzed during this study are included in this published article.

\section{Authors' contributions}

$\mathrm{MB}$ is the corresponding author and participated in the design of the case report. MD, NDV and EM were involved in acquisition of data and described the patient evolution and management. IMD, RCC and SR were involved in the analysis and interpretation of data, and revising the final manuscript. All authors confirm the authenticity of all the raw data. All authors have read and approved the final manuscript.

\section{Ethics approval and consent to participate}

Ethics approval was obtained from the Medical Ethics Commission for Clinical Studies in the Constanta Clinical Hospital of Infectious Diseases (approval no. 6/24.05.2021).

\section{Patient consent for publication}

Written informed consent was obtained from the patient prior to publication at the time of admission.

\section{Competing interests}

The authors declare that they have no competing interests.

\section{References}

1. Momtazmanesh S, Shobeiri P, Hanaei S, Mahmoud-Elsayed H, Dalvi B and Malakan EM: Cardiovascular disease in COVID-19: A systematic review and meta-analysis of 10,898 patients and proposal of a triage risk stratification tool. Egypt Heart J 72: 41, 2020.
2. Hessami A, Shamshirian A, Heydari K, Pourali F, Alizadeh-Navaei R, Moosazadeh M, Abrotan S, Shojaie L, Sedighi S, Shamshirian D and Rezaei N: Cardiovascular diseases burden in COVID-19: Systematic review and meta-analysis. Am J Emerg Med 46: 382-391, 2020.

3. Linschoten M, Peters S, van Smeden M, Jewbali LS, Schaap J, Siebelink HM, Smits PC, Tieleman RG, van der Harst P, van Gilst WH, et al: Cardiac complications in patients hospitalised with COVID-19. Eur Heart J Acute Cardiovasc Care 9: $817-823,2020$

4. Li JS, Sexton DJ, Mick N, Nettles R, Fowler VG Jr, Ryan T, Bashore T and Corey GR: Proposed modifications to the duke criteria for the diagnosis of infective endocarditis. Clin Infect Dis 30: 633-638, 2000.

5. Ramos-Martínez A, Fernández-Cruz A, Domínguez F, Forteza A, Cobo M, Sánchez-Romero I and Asensio A: Hospital-acquired infective endocarditis during Covid-19 pandemic. Infect Prev Pract 2: 100080, 2020.

6. Erdem H,Puca E, Ruch Y, Santos L, Ghanem-Zoubi N, Argemi X, Hansmann Y, Guner R, Tonziello G, Mazzucotelli JP, et al: Portraying infective endocarditis: Results of multinational ID-IRI study. Eur J Clin Microbiol Infect Dis 38: 1753-1763, 2019.

7. Hayes DE, Rhee DW, Hisamoto K, Smith D, Ro R, Vainrib AF, Bamira D, Zhou F and Saric M: Two cases of acute endocarditis misdiagnosed as COVID-19 infection. Echocardiography 38: 798-804, 2021.

8. Amir M, Djaharuddin I, Sudharsono A and Ramadanyd S: COVID-19 concomitant with infective endocarditis: A case report and review of management. Int J Infect Dis 98: 109-112, 2020.

9. Gurghean AL, Savulescu-Fiedler I and Mihailescu A: Multiple cardiac complications after adjuvant therapy for breast cancer: The importance of echocardiography. A case report and review of the literature. Med Ultrason 19: 117-120, 2017.

10. Streinu-Cercel A, Săndulescu O, Poiană C, Dorobanţu M, Mircescu G, Lăzureanu VE, Dumitru IM and Chirilă O, Streinu-Cercel A; Extended Consensus Group: Consensus statement on the assessment of comorbidities in people living with HIV in Romania. Germs 9: 198-210, 2019.

11. Dumitru IM, Lilios G and Arbune M: Respiratory infections and air pollution, retrospective study over the past 10 years. J Environ Prot Ecol 19: 1445-1451, 2018.

12. Calina D, Docea AO, Petrakis D, Egorov AM, Ishmukhametov AA, Gabibov AG, Shtilman MI, Kostoff R, Carvalho F, Vinceti M, et al: Towards effective COVID-19 vaccines: Updates, perspectives and challenges (Review). Int J Mol Med 46: 3-16, 2020.

13. Docea AO, Tsatsakis A, Albulescu D, Cristea O, Zlatian O, Vinceti M, Moschos SA, Tsoukalas D, Goumenou M, Drakoulis N, et al: A new threat from an old enemy: Re-emergence of coronavirus (Review). Int J Mol Med 45: $1631-1643,2020$ 